ARTICLE

Received 3 Jan 2014 | Accepted 27 Mar 2014 | Published 7 May 2014

DOI: $10.1038 /$ ncomms4731

\title{
Pressure-induced semiconducting to metallic transition in multilayered molybdenum disulphide
}

\author{
Avinash P. Nayak ${ }^{1, \star}$, Swastibrata Bhattacharyya ${ }^{2, \star},{\text { Jie } Z \mathrm{Zu}^{3,4}, \text { Jin Liu }^{3}, \text { Xiang Wu }{ }^{3} \text {, Tribhuwan Pandey }}^{2}$, \\ Changqing $\mathrm{Jin}^{4}$, Abhishek K. Singh ${ }^{2}$, Deji Akinwande ${ }^{1} \&$ Jung-Fu Lin ${ }^{3}$
}

Molybdenum disulphide is a layered transition metal dichalcogenide that has recently raised considerable interest due to its unique semiconducting and opto-electronic properties. Although several theoretical studies have suggested an electronic phase transition in molybdenum disulphide, there has been a lack of experimental evidence. Here we report comprehensive studies on the pressure-dependent electronic, vibrational, optical and structural properties of multilayered molybdenum disulphide up to $35 \mathrm{GPa}$. Our experimental results reveal a structural lattice distortion followed by an electronic transition from a semiconducting to metallic state at $\sim 19 \mathrm{GPa}$, which is confirmed by ab initio calculations. The metallization arises from the overlap of the valance and conduction bands owing to sulphur-sulphur interactions as the interlayer spacing reduces. The electronic transition affords modulation of the opto-electronic gain in molybdenum disulphide. This pressuretuned behaviour can enable the development of novel devices with multiple phenomena involving the strong coupling of the mechanical, electrical and optical properties of layered nanomaterials.

\footnotetext{
${ }^{1}$ Department of Electrical and Computer Engineering, The University of Texas at Austin, Austin, Texas 78712, USA. ${ }^{2}$ Materials Research Center, Indian Institute of Science, Bangalore 560-012, India. ${ }^{3}$ Department of Geological Sciences, The University of Texas at Austin, Austin, Texas 78712, USA. ${ }^{4}$ Beijing National Laboratory for Condensed Matter Physics, Institute of Physics, Chinese Academy of Sciences, Beijing 100190, China. ${ }^{*}$ These authors contributed equally to this work. Correspondence and requests for materials should be addressed to D.A. (email: deji@ece.utexas.edu).
} 
$\mathrm{R}$ ecent advances in the preparation of atomically thin layers of van der Waals (vdW) bonded crystalline solids by mechanical exfoliation or chemical synthesis have allowed for renewed investigations of two-dimensional (2D) materials beyond graphene but with a similar layered hexagonal structure $^{1-5}$. Within this class of 2D materials are transition metal dichalcogenides (TMDs), such as molybdenum disulphide $\left(\mathrm{MoS}_{2}\right)$, which have been increasingly explored to access a wealth of phenomena including opto-electronics ${ }^{5}$, valleytronics ${ }^{6}$, spintronics $^{7}$ and coupled electro-mechanics ${ }^{8}$. All of these phenomena arise from the complex thickness-dependent physical and electronic structures of the multilayered materials, in which the coupled electro-mechanics can be regarded as straintronics. Multilayered $\mathrm{MoS}_{2}$, having a band gap $\left(E_{\mathrm{g}}\right)$ of $\sim 1.2 \mathrm{eV}^{1}$, is composed of stacked tri-atomic sheets where each tri-atomic monolayer exhibits a sandwiched structure with a plane of transition metal molybdenum atoms covalently bonded to, and sandwiched between, two planes of chalcogen sulphur atoms (Fig. 1a). Unlike monoatomic multilayered graphene with $s p^{2}$ hybridization, the diatomic composition of multilayered $\mathrm{MoS}_{2}$ coupled with its $d$-orbital electronic states ${ }^{9}$ and the small $6.5 \AA$ $\mathrm{vdW}$ interlayer gap raises the prospects of strong sulphursulphur interlayer interactions under axial compression that might lead to an electronic phase transition.

Previously, several approaches have been undertaken to alter the solid-state properties of $\mathrm{MoS}_{2}$, including chemical doping $^{3,10-12}$, intercalation ${ }^{13,14}$, surface functionalization ${ }^{15}$ and defect engineering ${ }^{11,16,17}$. Furthermore, previous theoretical studies have suggested that an iso-structural semiconducting to metallic (S-M) transition in multilayered $\mathrm{MoS}_{2}$ can be induced by pressure or strain ${ }^{18-20}$. Despite the broad interest in this phenomenon in layered nanomaterials, previous experimental results only reported a limited reduction in the electrical resistance ${ }^{17,21-23}$ with no clear evidence for the pressureinduced S-M transition.

In the present study, we have investigated high-purity exfoliated single crystalline $\mathrm{MoS}_{2}$ with a stoichiometric chemical composition to evaluate the structural, vibrational, electrical and optical dependence at pressures up to $35 \mathrm{GPa}$. These results show a lattice distortion involving anisotropic $c / a$ axial compression beginning at $\sim 10 \mathrm{GPa}$ in multilayered $\mathrm{MoS}_{2}$ leading to an intermediate state (IS) followed by a pressure-induced S-M transition at $\sim 19 \mathrm{GPa}$. First-principle theoretical calculations, which are performed to shed light on the underlying physics responsible for the S-M transition, attribute the origin of the metallic electronic states to sulphur-sulphur interactions as the vdW gap closes at high pressures. Our combined experimental and theoretical results here open a new window of opportunity for the development of nanoscale pressure switches, sensors and multi-physics devices with coupled electrical, vibrational, optical and structural properties using multilayered $\mathrm{MoS}_{2}$ and semiconducting TMDs.

\section{Results}

Diamond anvil cell. In this work, we have explored applied pressure effects via a high-pressure diamond anvil cell (DAC), as a controllable and reversible degree of freedom to modulate the properties of multilayered single-crystal $\mathrm{MoS}_{2}$. The DAC technique coupled with a hydrostatic pressure medium (Fig. 1b) has been shown to be effective in elucidating the electrical, vibrational, optical and structural properties of a plethora of materials $\mathrm{s}^{21,23-25}$. The optically transparent window of the diamond allows us to conduct a series of in situ highpressure experiments including accurate pressure calibration from ruby fluorescence, electrical transport measurements and optical Raman and synchrotron X-ray diffraction (XRD) spectroscopy ${ }^{26}$, leading to the observation of S-M transition in multilayered $\mathrm{MoS}_{2}$ at high pressures. Pristine multilayered crystalline $\mathrm{MoS}_{2}$ was transferred onto the DAC using micron-edged tweezers for investigations of the electronic, phononic and structural behaviours at pressures up to $35 \mathrm{GPa}$ (see Methods for details). In addition, first-principle theoretical calculations were performed to elucidate the underlying material physics of the transition.
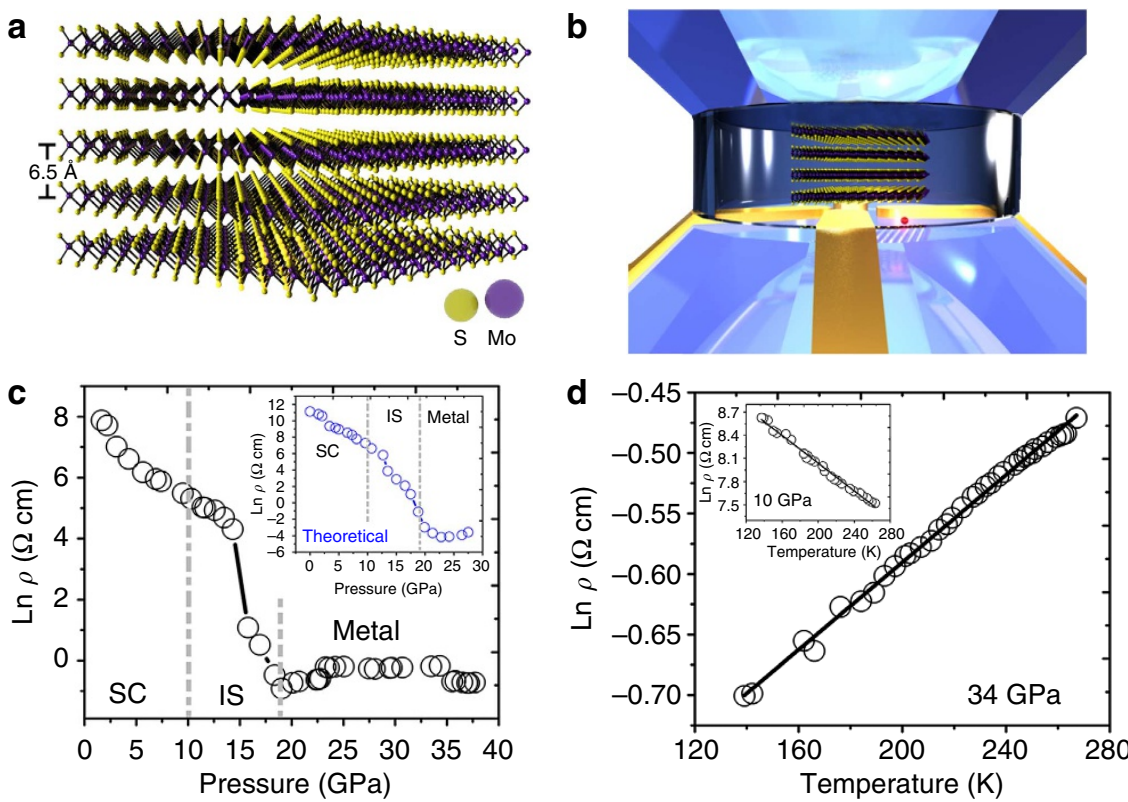

Figure 1 | Experimental setup and the electronic properties of multilayered $\mathbf{M o S}_{\mathbf{2}}$. (a) The cross-section illustration of multilayered MoS 2 with an interlayer (c axis) spacing of $\sim 6.5 \AA$. (b) A 3D illustration of multilayered $\mathrm{MoS}_{2}$ in a DAC pressure medium for compression experiments. (c) Pressuredependent electrical resistivity of $\mathrm{MoS}_{2}$. Three characteristic regions have been identified: semiconducting (SC), intermediate state (IS) and metallic regions. Inset: theoretically calculated pressure-dependent electrical resistivity. (d) Temperature-dependent resistivity of $\mathrm{MoS}_{2}$ in the metallic state. Inset: the experimental temperature-dependent semiconducting behaviour of $\mathrm{MoS}_{2}$. The solid lines serve as visual guides. 
Electrical conductivity and electronic structures. In situ electrical conductivity measurements at room temperature using standard four-point metallic contacts reveal a gradual decrease in resistivity $(\rho)$ with pressure up to $10 \mathrm{GPa}$, which can be understood in the context of a pressure-activated carrier transport model in good agreement with the experimental data (see Supplementary Note 1 and Supplementary Fig. 1). Between 10-19 $\mathrm{GPa}$ an abrupt decrease of about three orders of magnitude (Fig. 1c) is observed. Theoretical $a b$ initio band structure calculations of the multilayer $\mathrm{MoS}_{2}$ corroborates the experimental result with a predicted transition at $\sim 20 \mathrm{GPa}$ (Fig. 1c, inset), which was theoretically found to correspond to a vanishing band gap owing to a S-M electronic transition.

In addition, in situ temperature-dependent resistivity measurements were performed in the DAC in order to determine the resistivity profiles before and after the transition. It is well known from classical solid-state physics that resistivity decreases with temperature $(T)$ for semiconductors owing to thermally activated carriers, in contrast to increasing resistivity for metallic conductors due to increased electron-phonon scattering ${ }^{27}$. Our resistivity measurements for multilayered $\mathrm{MoS}_{2}$ reflect the aforementioned classical semiconducting behaviour as indicated by the negative temperature coefficient of resistivity at $10 \mathrm{GPa}$ (Fig. 1d, inset) for the semiconducting state, whereas a positive temperature coefficient of $\rho$ is observed at $34 \mathrm{GPa}$ for the metallic state (Fig. 1d). Moreover, the profile of the temperaturedependent resistivity magnitude shown in Fig. 1d follows an exponential decreasing trend at $10 \mathrm{GPa}$ and a linear $(\rho \alpha T)$ trend at $34 \mathrm{GPa}$ consistent with the classical behaviours of semiconducting and metallic materials respectively. Calculations using Boltzmann transport theory (BTT) for the temperature-dependent resistivity at $\sim 10 \mathrm{GPa}$ and $35 \mathrm{GPa}$ support these experimental findings (Supplementary Fig. 2).

Furthermore, theoretical calculations determine that the semiconducting nature of $\mathrm{MoS}_{2}$ originates from the fact that within a layer, Mo and $\mathrm{S}$ atoms are bound by mixed ionic and covalent bonding ${ }^{28}$. Under the application of pressure, the interlayer distance between the atomic planes decreases and the intra- as well as interlayer interactions are expected to alter the electronic properties of $\mathrm{MoS}_{2}$. Along the [0001] direction, $\mathrm{MoS}_{2}$ with anisotropic linear incompressibilities will experience maximum compression, that is, the interlayer distance ( $c$ axis) decreases more significantly than the intralayer distance ( $a$ axis). The hydrostatic pressure causes in- and out-of-plane strain. In order to analyse the effect of in-plane strain in achieving the S-M transition, we calculate the charge density redistribution keeping the in-plane strain constant, while varying the out-of-plane strain. Figure 2a,d shows the charge redistribution for a constant out-of-plane strain $(6.9 \%)$ but at a different in-plane strain of 2.2 and $5.2 \%$, respectively. Figure 2b,c shows the same but at a higher out-of-plane strain (11\%). It can be seen that the interlayer interaction is enhanced with the increasing in-plane strain. Although, the S-M transition is caused by interlayer interaction due to the normal or out-of-plane strain, the in-plane strain helps to achieve this transition at a lower pressure.

With increasing pressure, our theoretical calculations show that the electronic charge moves away from the $\mathrm{S}$ atoms and accumulates onto the Mo atoms. Near the critical transition pressure, the maximum charge redistribution is predicted to occur in between the $\mathrm{MoS}_{2}$ layers across the vdW gap, indicating enhanced S-S interaction. The change in the interlayer interaction from a weak vdW to a stronger interaction near the critical pressure is also supported by the derived lattice parameters using $\mathrm{XRD}$ spectra. However, these results do not give information regarding the orbitals involved in the charge transfer. Therefore, theoretical calculations for the angular momentum resolved density of states (LDOS) as a function of pressure were also performed to understand the contribution from the different orbitals of Mo and S atoms (Supplementary Fig. 3). a
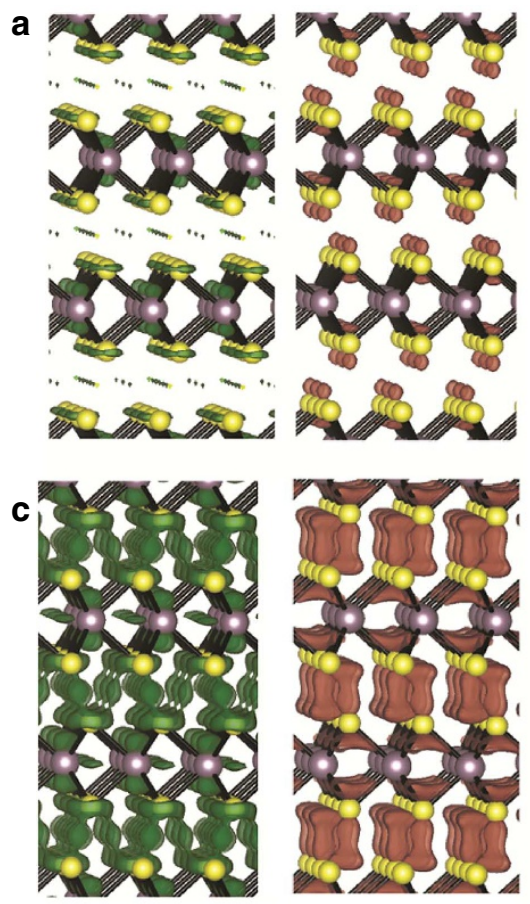

b

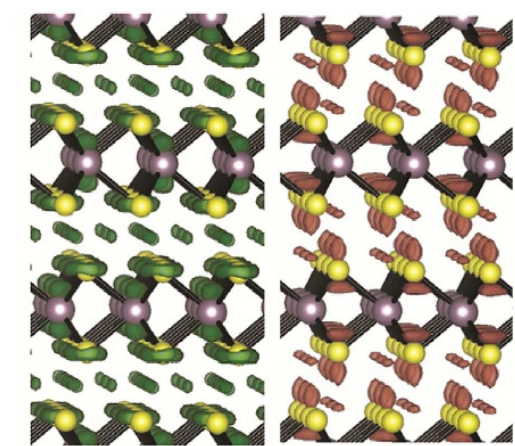

d

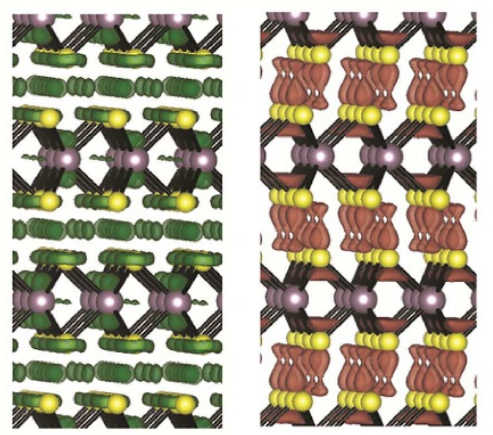

Figure 2 | Theoretical charge density isosurfaces of multilayered $\mathbf{M o S}_{\mathbf{2}}$. Charge accumulation (green) and depletion (orange) at the (a) in-plane and out-of-plane strains of 2.2 and $6.9 \%$, respectively, (b) in-plane and out-of-plane strains of 2.2 and $11 \%$, respectively, and (c) in-plane and out-of-plane strains of 5.2 and 11\%, respectively. (d) Same in-plane strain as in (c) but with $6.9 \%$ out-of-plane strain. 
With increasing pressure, the contribution to the conduction band minima (CBM) from S- $p_{x}$ and $\mathrm{S}-p_{y}$ orbitals decreases, while the contribution from S- $p_{z}$ orbitals increases. Similarly, there is a reduction with increasing pressure in contributions from Mo- $p$ orbitals, while the contribution from Mo- $d_{z}^{2}$ increases.

Optical Raman vibrational spectroscopy. Raman spectroscopy is considered to be a very accurate tool for gaining insight on the lattice vibrations of crystalline materials and has been commonly used to characterize the two prominent vibrational modes $\left(\mathrm{E}_{2 \mathrm{~g}}\right.$ and $A_{1 g}$ ) that are accessible in $\mathrm{MoS}_{2}$ (refs 29-31). In order to elucidate the S-M electronic transition, Raman measurements were conducted in situ to probe vibrational changes in the multilayered $\mathrm{MoS}_{2}$ under high pressure. It is well known that inducing pressure in $\mathrm{MoS}_{2}$ results in an increase in the Raman shift (Fig. 3a) similar to other layered materials ${ }^{30,32}$. Analyses of the high-pressure Raman spectra reveal that the $\mathrm{A}_{1 \mathrm{~g}}$ mode shows three distinct regions (Fig. 3b): (i) significantly increasing Raman shift up to about $10 \mathrm{GPa}$, (ii) nearly invariant shift with pressure between $10 \mathrm{GPa}$ and $19 \mathrm{GPa}$ and (iii) pressure-dependent Raman shift above $19 \mathrm{GPa}$. An evaluation of the Raman intensity ratio (between the $\mathrm{E}_{2 \mathrm{~g}}$ and $\mathrm{A}_{1 \mathrm{~g}}$ ) also reveals three distinct regions with the maximum intensity ratio observed in the metallic region (Supplementary Fig. 4). We label these regions starting from low to high pressures as the semiconducting (SC), intermediate state (IS), and metallic regions respectively. In light of these observations, the out-of-plane vibrational mode $\left(\mathrm{A}_{1 \mathrm{~g}}\right)$ can be considered the more prominent Raman mode that is correlated with the semiconducting to metallic transition in $\mathrm{MoS}_{2}$, in contrast to the in-plane mode $\left(\mathrm{E}_{2 \mathrm{~g}}\right.$ mode) that shows a less distinct character across the transition. The IS can be understood within the context of phonon softening (PS), which describes the electron and $\mathrm{A}_{1 \mathrm{~g}}$ phonon interaction where inter-valence charge transfer between the $\mathrm{MoS}_{2}$ layers plays an important role in the structural distortion of the crystal symmetry ${ }^{33}$. Several materials have been previously reported to exhibit an intermediate state with soften phonon behaviour while undergoing a transition from a semiconductor to a metallic state $\mathrm{s}^{34,35}$ or when stress or strain is applied $^{36,37}$. In fact, materials that exhibit superconductivity at high transition temperatures $\left(T_{\mathrm{c}}\right)$ are often associated with structural distortions that are characterized by PS subsequently resulting in either a structural or electronic phase transition ${ }^{38}$. In our study here, the intermediate state ranges from $\sim 10 \mathrm{GPa}$ to $19 \mathrm{GPa}$ where both the Raman shift for the $\mathrm{A}_{1 \mathrm{~g}}$ mode (Fig. $3 \mathrm{~b}$ ) and the spacing between the two modes (Fig. 3c) show little dependence on pressure suggesting that the lattice structure is distorted. Moreover, the full-width at half-maximum (FWHM) of the two modes initially increases with pressure followed by a subsequent sharp drop of $\sim 19$ GPa (Fig. $3 d$ ). It should be noted that in a recent report, a drop in the FWHM was also observed during a phase transition in $\mathrm{Sb}_{2} \mathrm{Se}_{3}$, a topological insulator that exhibits a metallic state at the surface ${ }^{39}$.

The effect of pressure on the vibrational properties of $\mathrm{MoS}_{2}$ can be further understood using theoretically calculated phonon dispersion curves based on the Perdew-Burke-Ernzerhof (PBE) functional method. We note that the calculated dispersion curves are consistent with that calculated using the local density approximation $^{26}$, justifying the use of the method. Analysis of the $A_{1 g}$ and $E_{2 g}$ modes as a function of pressure (which can be identified at the calculated $\Gamma$ point as shown in Supplementary Fig. 5) reveal that the splitting in the bands increases with applied pressure, resulting in significant changes in the Raman frequencies of the two modes (Fig. 3b). a
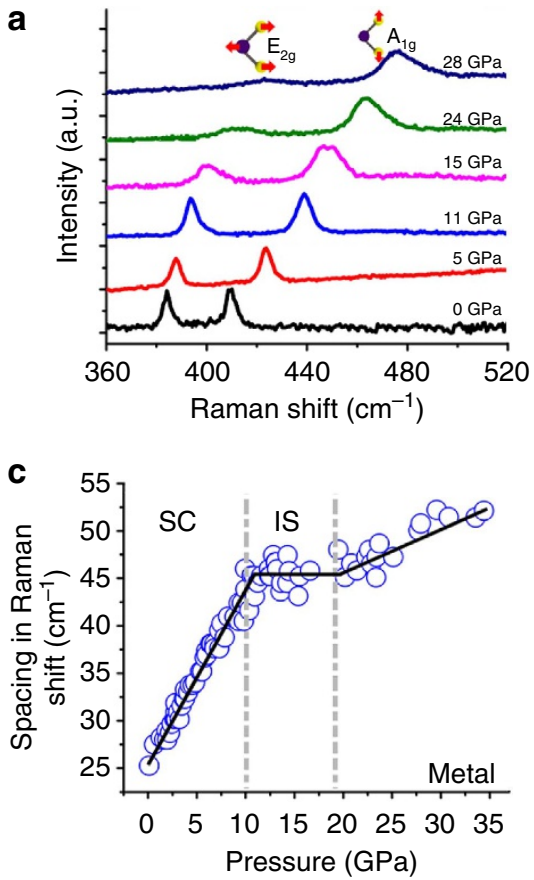

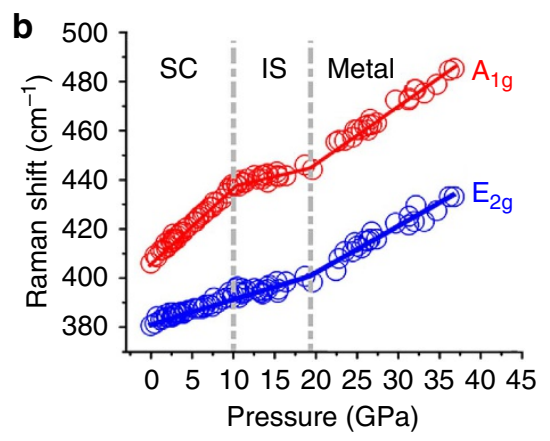

d

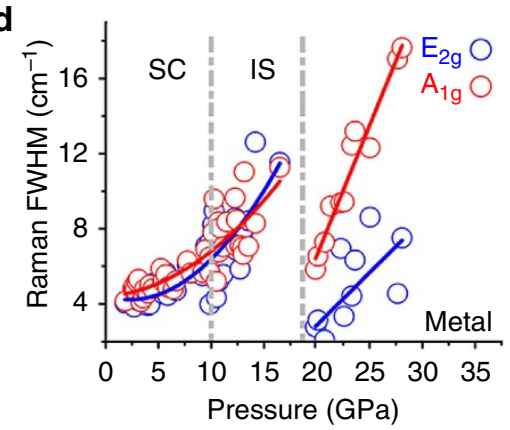

Figure 3 | Raman spectroscopic results of $\mathbf{M o S}_{\mathbf{2}}$ at high pressures. (a) Raman spectra at representative pressure points. The inset is an illustration of the in-plane and out-of-plane vibrations of the two key modes $E_{2 g}$ and $A_{1 g}$, respectively. (b) The Raman shifts of the two modes with increasing pressures. The solid red and blue lines serve as visual guides. (c) The difference in Raman frequencies between the $A_{1 g}$ and $E_{2 g}$ modes as a function of pressure flattens out in the IS state and rises at a smaller slope in the metallic region. The solid black line is a visual guide. (d) The FWHM for the $A_{1 g}$ and $\mathrm{E}_{2 \mathrm{~g}}$ Raman modes across the electronic transition. A sharp drop is observed at $\sim 19 \mathrm{GPa}$ where $\mathrm{MoS}_{2}$ transitions from the IS to a metallic phase.

The solid red and blue lines serve as visual guides for the $A_{1 g}$ and $E_{2 g}$ modes, respectively. 
Opto-electronic properties. Photoconductivity measurements have also been performed as a complementary means for understanding the opto-electronic properties of materials, which is dependent on the material's band gap. To provide perspective, the pressure-dependent band gap for multilayered $\mathrm{MoS}_{2}$ (Fig. 4a) can be understood from theoretical analysis using the PBE functional under the generalized gradient approximation (GGA). We note that the calculated unstrained $E_{\mathrm{g}}$ of $1.03 \mathrm{eV}$ is $\sim 16 \%$ less than the experimental value ${ }^{40}$. This underestimation of the bandgap is a well-known problem due to the presence of artificial self-interaction and the absence of the derivative discontinuity in the exchange-correlation potential with the PBE/GGA methods ${ }^{41}$. Hybrid Heyd-Scuseria-Ernzerhof ${ }^{42}$ functional and DFT with many-body perturbation theory in the GW approximation ${ }^{43}$ are among the few methods to correctly predict the band gap of TMDs; however, these methods are computationally very expensive. Nevertheless, our previous study for bilayer $\mathrm{MoS}_{2}$ has shown that the overall nature of the variation in the $E_{\mathrm{g}}$ and the band structure with pressure are independent of the functional used ${ }^{18}$ and that the PBE functional gives reasonably accurate results, justifying the use of the PBE/GGA methods for this work. With the increase in pressure, the degeneracy in the bands of the unstrained structure is lifted due to the enhanced interlayer interaction. The split bands in the valence band maxima (VBM) at the $\Gamma$ point and the CBM between the $\mathrm{K}$ and $\Gamma$ point start to move toward the Fermi level. The $E_{\mathrm{g}}$ reduces consequently and becomes metallic when the CBM crosses the Fermi level at a pressure of $23.8 \mathrm{GPa}$ (Fig. 4a), which correlates well with the transition pressures of $\sim 19 \mathrm{GPa}$ in the experimental resistivity measurements and Raman spectra. Analyses of the band structure in the metallic state indicate the overlap of the conduction and valence bands at the Fermi level (Fig. 4b).
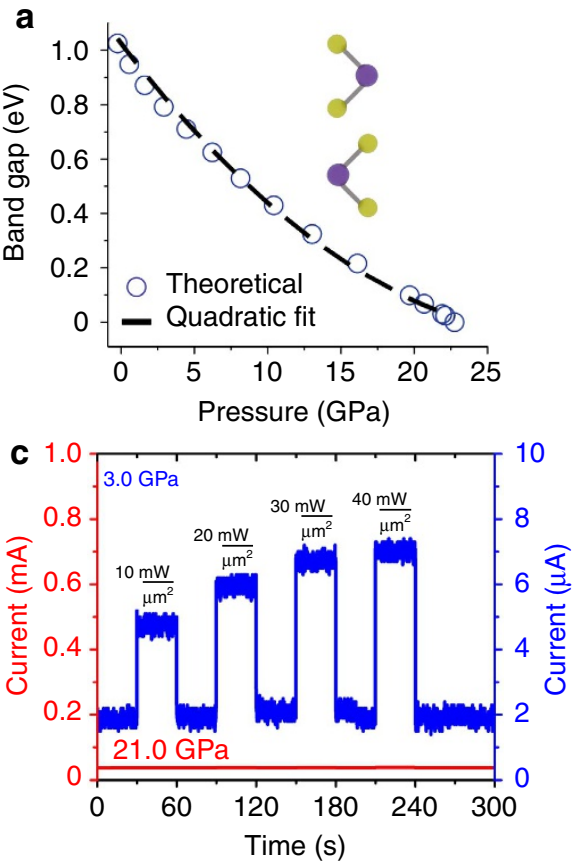

Unlike metals, semiconductors show an increase in the electrical conductivity when illuminated by light, and are therefore considered photoconductive. In particular, semiconducting $\mathrm{MoS}_{2}$ has been found to offer sufficient photo-response that is suitable for practical photoconductive applications ${ }^{3-5,44}$. Opto-electronics require electron-hole generation/recombination, whereas the opto-electronic response of $\mathrm{MoS}_{2}$ under green laser illumination $(2.3 \mathrm{eV})$ in the event of a semiconducting to metallic transition in $\mathrm{MoS}_{2}$ is expected to be significantly diminished in the metallic state (Fig. 4c). As a result, the photocurrent gain (ratio of current under illumination to dark current) was found to decrease with pressure as shown in Fig. 4d. The current gain decreases at a linear rate $\left(\Delta I_{\text {gain }} / \Delta \mathrm{GPa}=-7.5 \% / \mathrm{GPa}\right)$ until $\sim 10$ $\mathrm{GPa}$ where the current gain saturates to unity, indicating that photo-response is vanishing as the band gap begins to close.

XRD and HR-TEM studies. Electronic phase transitions can be accompanied by a structural or iso-structural phase transition. In order to clarify potential structural changes at high pressure, in situ high-pressure synchrotron XRD experiments were conducted to probe the perturbation of the crystal structure of multilayered $\mathrm{MoS}_{2}$ under hydrostatic pressure. Two independent in situ high-pressure angle-dispersive XRD experimental runs were carried out at room temperature using a symmetric DAC. For the entire experimental pressure range, all the diffraction peaks can be refined to the hexagonal $P 6_{3} / m m c$ structure using the Rietveld refinement ${ }^{45}$. On the basis of the hexagonal structure model, the crystal lattice parameters for $\mathrm{MoS}_{2}$ from 0 to $28 \mathrm{GPa}$ were calculated (Fig. 5a). The structure of the bulk unstrained $\mathrm{MoS}_{2}$ can be viewed as ABAB stacked single layers of the material $^{22}$. Though we did not observe any new peak(s) for multilayered $\mathrm{MoS}_{2}$ up to $24 \mathrm{GPa}$ in the XRD, a change along the

b
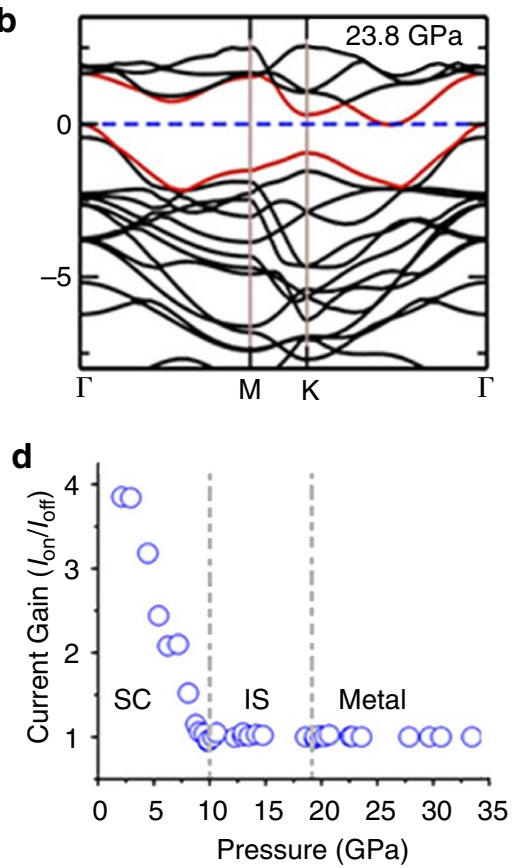

Figure 4 | Theoretical and experimental pressure-dependent opto-electronic properties of $\mathbf{M o S}_{\mathbf{2}}$. (a) Theoretical calculation of the pressure-dependent band gap of multilayered $\mathrm{MoS}_{2}$. The bandgap-pressure dependence can be modelled as a quadratic function, $E_{g}=E_{g o}+a P+b P^{2}$ was used, where $a=-70 \mathrm{meV} \mathrm{GPa}^{-1}$ and $b=1.13 \mathrm{meV} \mathrm{GPa}^{-2}$. Inset: the unit cell of $\mathrm{MoS}_{2}$. (b) Theoretical band structure of multilayered MoS 2 under hydrostatic pressure of 23.8 GPa. VBM and CBM are shown by red lines. (c) Optical switch behaviour before (blue lines at 3.0 GPa) and after (red line at $21.0 \mathrm{GPa}$ ) the S-M transition with various laser intensities. (d) Current gain (defined as $I_{\mathrm{on}} / I_{\text {off }}$ ratio) as a function of pressure when exposed to a $532 \mathrm{~nm}$ ( $2.3 \mathrm{eV}$ ) laser light with an intensity of $40 \mathrm{Wm}^{-2}$. 
a

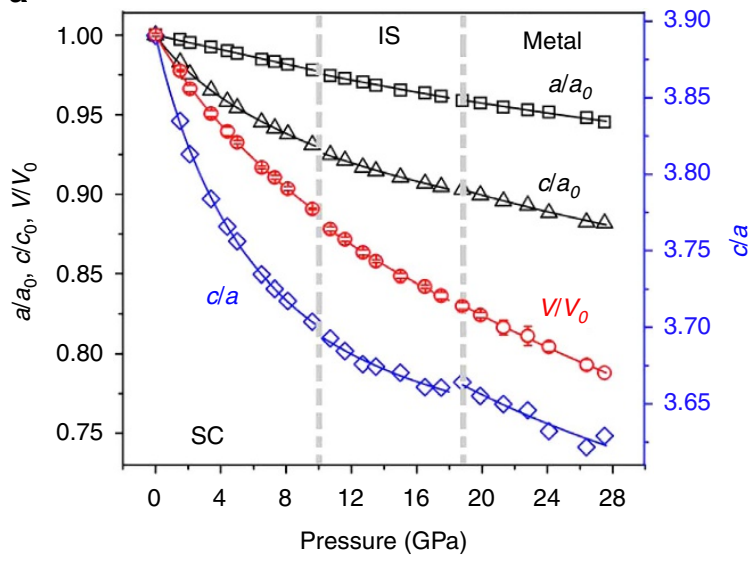

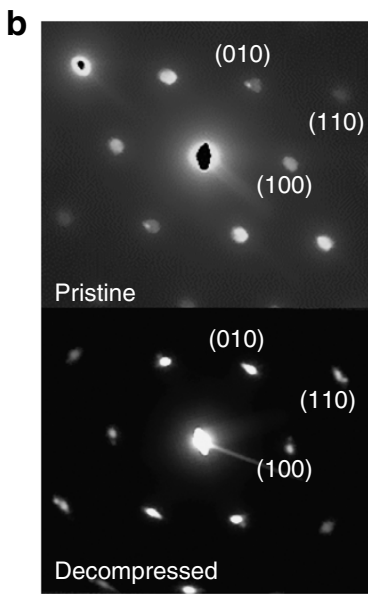

Figure 5 | Structural lattice parameters in multilayered $\mathbf{M o S}_{\mathbf{2}}$. (a) Experimental pressure dependence of the lattice parameters of $M o S_{2}$. The volume uncertainty bars are within $1 \%$ of the data. (b) The experimental SAED patterns of both pristine and decompressed MoS 2 . The patterns show that the single-crystal structure with the hexagonal symmetry was not significantly affected by high pressures and can be recovered back to its original crystal structure.

$c$ axis is observed at $\sim 19 \mathrm{GPa}$, which suggests that the ambient lattice structure of $\mathrm{MoS}_{2}$ is only weakly distorted. On the other hand, theoretical calculations were performed using the experimental lattice parameters as the reference, which are in close agreement with the GGA values. Results from theoretical pressure-dependent XRD calculations also confirm that there is no structural transition (Supplementary Fig. 6).

Characterizing the atomic structure and quality of $\mathrm{MoS}_{2}$ is critical for further understanding of the lattice distortion and electronic phase transition under pressure, and for ruling out the effects of lattice defects (that is, point defects, dislocations, grain boundaries and edges) that could play a role in influencing the experimental results ${ }^{11,16,17}$. For this purpose, high-resolution transmission electron microscopy (HR-TEM) and electron diffraction techniques were employed to evaluate the crystal structure of the same multilayered $\mathrm{MoS}_{2}$ before and after the pressure loading experiments. The morphology of the starting sample in HR-TEM shows an atomic $\mathrm{MoS}_{2}$ lattice having high crystallinity and the expected hexagonal lattice structure with a lattice spacing of $2.7 \AA$ corresponding to the [100] direction (Supplementary Fig. 7a). The cross-section HR-TEM analysis shows the layered structure of $\mathrm{MoS}_{2}$ having an interatomic $c$ axis spacing of $\sim 6.5 \AA$ (Supplementary Fig. 7b). Transmission electron microscopy energy-dispersive X-ray spectroscopy (TEM-EDX) analyses were performed on both the pristine and decompressed $\mathrm{MoS}_{2}$ to determine the stoichiometry, which was found to be a 1:2.07 ratio (Supplementary Fig. 7c), very close to the ideal 1:2 Mo:S ratio. Scanning electron microscopy (SEM) and scanning electron energy-dispersive X-ray spectroscopy (SEMEDX) for both pristine and decompressed samples also show negligible difference (Supplementary Fig. 7d). The fast Fourier transformation (FFT) of the HR-TEM image shows the reflection spots from (100), (010) and (110), while the selected area electron diffraction (SAED) patterns for both the pristine and decompressed sample indicate the crystallinity and hexagonal symmetry were also unaffected by the applied pressures (Fig. 5b). The crystallographic results indicate that the starting sample was basically recoverable back to its original crystal structure. Collectively, the HR-TEM, TEM-EDX, FFT, SEM-EDX and SAED results indicate that the sample was of high crystalline quality and purity, and maintained its original chemical composition and lattice structure after the pressure-loading experiments.

\section{Discussion}

Previous studies have shown that loading of a crystal lattice via strain or hydrostatic pressure can cause a sharp reduction in resistivity, which is a precursor for an $\mathrm{S}-\mathrm{M}$ transition $^{21}$. In agreement with prior theoretical studies ${ }^{18,19,46}$, our theoretically predicted band gap is found to depend inversely on pressure and that the critical pressure for the electronic phase transition is predicted to scale down for multilayered films from $\sim 16 \%$ normal compressive strain to $\sim 11 \%$ for bilayer $\mathrm{MoS}_{2}$ (Supplementary Fig. 8). For monolayer $\mathrm{MoS}_{2}$ this transition is predicted to occur at $\sim 10 \%$ bi-axial strain ${ }^{19}$. Reported studies on other materials such as $\mathrm{Sb}_{2} \mathrm{Te}_{3}$ (ref. 23), $\mathrm{WSe}_{2}$ (ref. 24), $\mathrm{BaNi}_{2} \mathrm{As}_{2}$ (ref. 38), FeS (ref. 47), $\mathrm{V}_{2} \mathrm{O}_{3}$ (ref. 48) and $\mathrm{VO}_{2}$ (ref. 49) have also shown a drop in resistivity before an electronic or structural phase transition in the material. These results have indicated that applying extremely high pressures onto such materials can even lead to a superconducting state. In $\mathrm{MoS}_{2}$, this drop in resistivity is correlated with the characteristic phonon modes $\left(\mathrm{E}_{2 \mathrm{~g}}\right.$ and $\left.\mathrm{A}_{1 \mathrm{~g}}\right)$, and concurrent with phonon softening in the intermediate state, which has been observed as a precursor for resistivity transition in other materials ${ }^{35-38}$. We find that both the valence band (VB) and the conduction band (CB) originate from the Mo- $d$ and S- $p$ orbitals with the VB having minor contributions from the Mo- $p$ orbital at zero pressure (Supplementary Fig. 3). With increasing pressure, the contribution from the $\mathrm{S}-p_{z}$ and Mo- $d_{z}^{2}$ orbitals increases in both $\mathrm{VB}$ and $\mathrm{CB}$, indicating a strong interlayer interaction. This analysis is in agreement with the charge redistribution. Experimentally, the bandgap can be extracted from temperature-dependent resistivity for intrinsic semiconductors; however, for the case of contemporary $\mathrm{MoS}_{2}$ materials, the prevailing n-type character prevents bandgap extraction directly from resistivity data.

We note that the $A_{1 g}$ mode arises from the out-of-plane vibration of the $S$ atoms with enhanced $S-S$ interactions as the $\mathrm{vdW}$ gap closes resulting in the observed metallization. In the intermediate state, vibrational phonon softening phenomenon in the Raman spectra has been studied extensively for other layered materials $^{39}$, suggesting that it can be used as a clear signature for the electronic phase transition in the multilayered $\mathrm{MoS}_{2}$. The pressure-dependent Raman frequency increase was observed to be higher for the out-of-plane $A_{1 g}$ mode than that of the in-plane $\mathrm{E}_{2 \mathrm{~g}}$ mode. This clearly indicates an enhancement of interlayer interactions with pressure. The multitude of pressure-dependent 
Raman spectra characteristics (FHWM, intensity ratio and peak spacing) are well correlated with the pressure-dependent resistivity, all showing an abrupt or particular response in the IS region. These strong correlations provide unambiguous experimental and theoretical support for the semiconducting to metallic phase transition in multilayered $\mathrm{MoS}_{2}$.

Since the neighbouring planes of the sulphur atoms along the $c$ axis are bonded by weak vdW forces, the $c$ axis contracts two times more than the $a$ axis below $28 \mathrm{GPa}$, exhibiting strong anisotropic compressional properties. The $c / a$ lattice-parameter ratio decreases monotonically with pressure up to $19 \mathrm{GPa}$, which indicates that the interactions between the adjacent planes of sulphur atoms become stronger under pressure. We note that the cla curve profile shows discontinuity at $\sim 19 \mathrm{GPa}$. Similar behaviour was also reported by Aksoy et al..$^{50}$ and can be attributed to a pressure-induced structural distortion in which $\mathrm{MoS}_{2}$ layers slide from the original $2 \mathrm{H}_{\mathrm{c}}-\mathrm{MoS}_{2}$ to the $2 \mathrm{H}_{\mathrm{a}}-\mathrm{MoS}_{2}$ structure $^{24}$. Based on theoretical studies by Hromadová et al. ${ }^{22}$, the peak positions in Raman measurements under high pressure coincide with the electronic properties of the emergent $2 \mathrm{H}_{\mathrm{a}}-\mathrm{MoS}_{2}$ phase. We thus conclude that the observed changes in the electrical and vibrational response can be solely attributed to an iso-structural electronic phase transition.

We have demonstrated for the first time that a drastic drop in resistivity can be attributed to an electronic phase transition from semiconducting to metallic in multilayered $\mathrm{MoS}_{2}$, which is further corroborated by in situ temperature-dependent resistivity measurement and Raman spectroscopy. Our results can be applied to novel $\mathrm{MoS}_{2}$ devices, where pressure can be an external stimulus to dynamically control the band gap and tune the optoelectronic response or photoconductive gain. The experimentally observed transition is in good agreement with ab initio theoretical calculations, allowing one to predict the thickness dependence of the critical transition pressure. Based on the similarity in the crystal and band structure of the semiconducting transitional metal dichalcogenides, the observed electronic phase transition in $\mathrm{MoS}_{2}$ is likely to be experimentally accessible in $\mathrm{MoSe}_{2}$, $\mathrm{WS}_{2}$ and $\mathrm{WSe}_{2}$ as predicted in a previous work ${ }^{18}$. The combined experimental results and theoretical insights indicate a new opportunity for the development of multi-physics device concepts with coupled mechanical, electrical and optical properties based on the tunable and unique material structure of the semiconducting transitional metal dichalcogenides.

\section{Methods}

Sample characterization. $\mathrm{MoS}_{2}$ single crystals were purchased from SPI Supplies (CAS\# 1317-33-5). The purity of the crystals was characterized and estimated to be $>99 \%$ by SPI supplies. The crystals were then thinned down to flakes of $\sim 150 \mathrm{~nm}$ thick and $100 \mu \mathrm{m}$ in length. Cross-section HR-TEM analyses of the sample flakes showed chemically and physically homogeneous layer surfaces at the nanometre scale. To understand the characteristics of the pristine as well as pressure-quenched samples (up to $\sim 35 \mathrm{GPa}$ and then quenched to ambient pressure), HR-TEM was used to analyse the lattice structures at the nanometre scale. Pristine $\mathrm{MoS}_{2}$ was exfoliated onto a Cu TEM grid (SPI\# 2040C-XA), while the quenched $\mathrm{MoS}_{2}$ was transferred onto the $\mathrm{Cu}$ TEM grid using a micro tip-needle for HR-TEM analyses. The beam spot size was $5 \mathrm{~nm}$ in the TEM-EDX and an aperture size of $160 \mathrm{~nm}$ was used for the selected area electron diffraction (SAED). HR-TEM analyses of the recovered samples from DAC compression experiments show that their crystallinity and hexagonal lattice structure have not been altered drastically by compression and decompression in the DAC (Fig. 5 and Supplementary Fig. 7).

High-pressure electrical resistance measurements. Four-point probe electrical technique was employed to measure the electrical resistivity of the single-crystal multilayered $\mathrm{MoS}_{2}$ as a function of pressure. Resistivity measurements as a func tion of temperature were also conducted in the SC, IS and metallic state. A modified short symmetric DAC having a pair of anvil culets of $400 \mu \mathrm{m}$ or $500 \mu \mathrm{m}$ was used in two separate runs to ensure reproducibility. A rhenium gasket was preindented from a thickness of $250 \mu \mathrm{m}$ to $40 \mu \mathrm{m}$ at $\sim 20 \mathrm{GPa}$, and a hole with a diameter of $200 \mu \mathrm{m}$ was drilled in the centre of the pre-indented area. Cubic BN (c-BN) fine powders were used as an insulating layer that was pressed into the pre-indented area and the drilled hole. Consequently, another hole of $100 \mu \mathrm{m}$ in diameter was drilled at the centre of the $200 \mu \mathrm{m}$ hole filled with c-BN, which was then further filled with soft h-BN fine powders as the pressure-transmitting medium while neon was used as the gas medium. A MoS 2 single crystal of $150 \mathrm{~nm}$ thick with dimensions of $\sim 100 \mu \mathrm{m} \times 266 \mu \mathrm{m}$ was loaded into the sample chamber together with three ruby spheres as the pressure calibrant and four Au electrodes with a length of $12.5 \mu \mathrm{m}$ for EC measurements. The resistivity of the sample was obtained by measuring the DC current changes in a pair of Au probes while sweeping DC voltages in another pair. The sample resistivity was derived by measuring the slope of the current-voltage $(I-V)$ plot after taking the sample thickness into account ${ }^{51}$. Quenched samples were further examined using crosssectional SEM for potential dimensional changes of the samples to ensure reliable EC correction. For the opto-electronic measurements under high pressure, a green laser with $532 \mathrm{~nm}$ wavelength $(2.3 \mathrm{eV})$ was focused down to a $10 \mu \mathrm{m} \times 10 \mu \mathrm{m}$ window to ensure that the laser was exclusively on the sample.

High-pressure laser Raman spectroscopy. Raman spectra were measured using a green $532 \mathrm{~nm}$ Coherent Verdi V2 laser. The scattered light was dispersed by a 1,800 groves per mm grating and collected by an EMCCD (Andor Technology) resulting in a spectral resolution of $\sim 1 \mathrm{~cm}^{-1}$. The highest laser power level of $4 \mathrm{~mW}$ with a focused laser beam size of $10 \mu \mathrm{m}$ was used for the measurements to avoid potential over-heating or oxidation of the samples. Ruby fluorescence spectra were also collected using the same system for pressure calibrations; pressures and their uncertainties were determined from multiple measurements of the ruby spheres close to the sample in the sample chamber of the DAC before and after each Raman measurement.

High-pressure synchrotron XRD experiments. In situ high-pressure angle-dispersive XRD experiments were carried out at the High Pressure Collaborative Access Team (HPCAT) and GeoSoilEnviroCARS (GSECARS) of the Advanced Photon Source (APS), Argonne National Laboratory using a wavelength of $0.4066 \AA$ and $0.3344 \AA$, respectively, in a symmetric DAC. The diameter of the diamond culets was $400 \mu \mathrm{m}$ while the thickness of the sample loaded into the sample chamber was $\sim 20-25 \mu \mathrm{m}$. A Rhenium gasket pre-indented to $45 \mu \mathrm{m}$ thick with a drilled hole of $150 \mu \mathrm{m}$ in diameter was used as the sample chamber, while neon was used as the pressure-transmitting medium and the pressure was measured using a ruby fluorescence calibrant. XRD images were collected by an MAR CCD and were integrated using the Fit2D programme ${ }^{52}$. The integrated XRD patterns were further analysed using the GSAS programme package for the Rietveld refinement ${ }^{45}$. The solid line is the fitting result (Fig. 5a) using the third-order Birch-Murnaghan equation of state (BM EoS $)^{53,54}$ :

$$
P(\mathrm{GPa})=\frac{3}{2} \mathbf{K}_{0}\left[\left(\frac{V_{0}}{V}\right)^{\frac{7}{3}}-\left(\frac{V_{0}}{V}\right)^{\frac{5}{3}}\right]\left\{1-\left(3-\frac{3}{4} \mathbf{K}_{0}^{\prime}\right)\left[\left(\frac{V_{0}}{V}\right)^{\frac{7}{3}}-1\right]\right\},
$$

where $V$ and $V_{\mathbf{0}}$ represent the high-pressure and ambient-pressure unit cell volume, respectively; $\mathbf{K}_{0}$ and $\mathbf{K}_{0}^{\prime}$ refer to the bulk modulus and its pressure derivate at ambient conditions, respectively. The isothermal bulk modulus $K_{0}$ for the semiconducting state, IS state, and the metallic state was derived to be $57 \mathrm{GPa}$, $51 \mathrm{GPa}$ and $64 \mathrm{GPa}$ wih $K_{0}^{\prime}$ being $4.6 \mathrm{GPa}, 4.9 \mathrm{GPa}$ and $4.2 \mathrm{GPa}$ respectively.

First-principle theoretical calculations. Theoretical calculations were carried out using first-principle $a b$ initio DFT using Vienna $A b$ initio Simulation Package $(\mathrm{VASP})^{55}$. All-electron projector augmented wave potentials ${ }^{56,57}$ and the PBE ${ }^{58}$ GGA were used to account for the electronic exchange and correlation. The weak $\mathrm{vdW}$ interaction was incorporated by adding a semi-empirical dispersion potential (D) to the conventional Kohn-Sham DFT energy, through a pair-wise force field following Grimmes DFT-D2 method ${ }^{59}$. The crystal structure was optimized by employing a conjugate gradient scheme until the forces on every atom were minimized to be $<0.005 \mathrm{eV}^{-1}$, and that a well-converged Monkhorst-Pack $\mathbf{k}$-point set $(17 \times 17 \times 6)$ was used for this procedure. The phonon dispersion of $\mathrm{MoS}_{2}$ was calculated using density functional perturbation theory ${ }^{60}$ as implemented in VASP. An additional tool, Phonopy ${ }^{61}$, which supports the VASP interface, was used for extracting the phonon frequencies. The force-constants were calculated with a supercell of $2 \times 2 \times 2$ and the k-point set of $9 \times 9 \times 3$. The criterion for the force convergence used for the phonon calculations was $10^{-8} \mathrm{eV} \AA^{-1}$

Electrical conductivity of $\mathrm{MoS}_{2}$ is calculated using BTT within the constant scattering time approximation (CSTA $)^{27,62}$, as implemented in the BoltzTraP programme ${ }^{63}$. In the CSTA, it is assumed that the scattering time $(\tau)$ determining the electrical conductivity does not vary strongly with temperature. In the BTT, the motion of an electron is treated semi-classically. The group velocity of an electron in a particular band can be described as:

$$
v_{\alpha}(i, \mathbf{k})=\frac{1}{\hbar} \frac{\partial \varepsilon(i, \mathbf{k})}{\partial \mathbf{k}_{\alpha}}
$$

Where $\varepsilon(i, \mathbf{k})$ is the $i$ th energy band at point $\mathbf{k}$ and $\mathbf{k}_{\alpha}$ is $\alpha$ th component of wave-vector $\mathbf{k}$. 
From the group velocity $v_{\alpha}(i, \mathbf{k})$, the electrical conductivity tensor can then be calculated by the following equation:

$$
\sigma_{\alpha \beta}(T, \mu)=\frac{1}{V} \int \frac{e^{2}}{N} \sum \tau(i, \mathbf{k}) v_{\alpha}(i, \mathbf{k}) v_{\beta}(i, \mathbf{k}) \delta[\varepsilon-\varepsilon(i, \mathbf{k})]\left[-\frac{\partial f_{\mu}(T, \varepsilon)}{\partial \varepsilon}\right] \mathrm{d} \varepsilon
$$

where $\tau(i, \mathbf{k})$ is the relaxation time, $\alpha$ and $\beta$ are the Cartesian indices, $N$ is the total number of $\mathbf{k}$-points sampled, $V$ is the volume of the unit cell, $f_{\mu}$ is the Fermi Dirac distribution function and $e$ is the elementary charge.

In order to estimate the electrical transport properties of bulk $\mathrm{MoS}_{2}$, first the calculations for the electronic structure were performed using a denser $\mathbf{k}$-point set of $32 \times 32 \times 8$. This was followed by the calculation of group velocities by Fourier interpolation ${ }^{64}$ of the band energies as a function of $\mathbf{k}$. Consequently, the transport properties were calculated by using equation (3). From the electronic structure it is possible to calculate $\sigma / \tau$ as a function of temperature $(T)$ and carrier concentration $(n)$, but it is not possible to calculate $\sigma$ without relaxation time $(\tau)$. To approximate the relaxation time, we used experimental data from Mahalaway et al. ${ }^{65}$ The reported experimental electrical conductivity is $0.03 \Omega^{-1} \mathrm{~cm}^{-1}$ at room temperature, which combined with the calculated $\sigma / \tau$ yield $\tau=7.5 \times 10^{-14}$. We then calculate $\sigma$ as $\sigma / \tau \times \tau$.

\section{References}

1. Wang, Q. H. et al. Electronics and optoelectronics of two-dimensional transition metal dichalcogenides. Nature Nanotechnol. 7, 699-712 (2012).

2. Butler, S. Z. et al. Progress, challenges, and opportunities in two-dimensional materials beyond graphene. ACS Nano 7, 2898-2926 (2013).

3. Fontana, M. et al. Electron-hole transport and photovoltaic effect in gated $\mathrm{MoS}_{2}$ Schottky junctions. Sci. Rep. 3, 1634 (2013).

4. Yin, Z. et al. Single-layer $\mathrm{MoS}_{2}$ phototransistors. ACS Nano 6, 74-80 (2011)

5. Lee, H. S. et al. $\mathrm{MoS}_{2}$ nanosheet phototransistors with thickness-modulated optical energy gap. Nano Lett. 12, 3695-3700 (2012).

6. Behnia, K. Condensed-matter physics: polarized light boosts valleytronics. Nat. Nanotechnol. 7, 488-489 (2012).

7. Zeng, H. et al. Valley polarization in $\mathrm{MoS}_{2}$ monolayers by optical pumping. Nat. Nanotechnol. 7, 490-493 (2012).

8. Ghorbani-Asl, M. et al. Electromechanics in $\mathrm{MoS}_{2}$ and $\mathrm{WS}_{2}$ : nanotubes versus monolayers. Sci. Rep. 3, 2961 (2013).

9. Splendiani, A. et al. Emerging photoluminescence in monolayer $\mathrm{MoS}_{2}$. Nano Lett. 10, 1271-1275 (2010).

10. Sachs, B. et al. Doping mechanisms in graphene- $\mathrm{MoS}_{2}$ Hybrids. Appl. Phys. Lett. 103, 251607 (2013).

11. Ataca, C., Sahin, H., Aktürk, E. \& Ciraci, S. Mechanical and electronic properties of $\mathrm{MoS}_{2}$ nanoribbons and their defects. J. Phys. Chem. C 115, 3934-3941 (2011).

12. Radisavljevic, B. \& Kis, A. Mobility engineering and a metal-insulator transition in monolayer $\mathrm{MoS}_{2}$. Nat. Mater. 12, 815-820 (2013).

13. Tongay, S. et al. Thermally driven crossover from indirect toward direct bandgap in 2D semiconductors: $\mathrm{MoSe}_{2}$ versus $\mathrm{MoS}_{2}$. Nano Lett. 12, 5576-5580 (2012).

14. Eda, G. et al. Photoluminescence from chemically exfoliated $\mathrm{MoS}_{2}$. Nano Lett. 11, 5111-5116 (2011).

15. Ataca, C. \& Ciraci, S. Functionalization of single-layer $\mathrm{MoS}_{2}$ honeycomb structures. J. Phys. Chem. C 115, 13303-13311 (2011).

16. Qiu, H. et al. Hopping transport through defect-induced localized states in molybdenum disulphide. Nat Commun. 4, 2642 (2013).

17. Fu, D. et al. Mechanically modulated tunneling resistance in monolayer $\mathrm{MoS}_{2}$. Appl. Phys. Lett. 103, 183105-1-183105-3 (2013).

18. Bhattacharyya, S. \& Singh, A. K. Semiconductor-metal transition in semiconducting bilayer sheets of transition-metal dichalcogenides. Phys. Rev. B 86, 075454-075454 (2012).

19. Johari, P. \& Shenoy, V. B. Tuning the electronic properties of semiconducting transition metal dichalcogenides by applying mechanical strains. ACS Nano 6, 5449-5456 (2012).

20. Wei, L. et al. $\mathrm{MoS}_{2}$. Phys. B: Condens. Matter. 405, 2498-2502 (2010).

21. Dave, M. et al. High pressure effect on $\mathrm{MoS}_{2}$ and $\mathrm{MoSe}_{2}$ single crystals grown by CVT method. Bull. Mater. Sci. 27, 213-216 (2004).

22. Hromadová, L. et al. Structure change, layer sliding, and metallization in highpressure $\mathrm{MoS}_{2}$. Phys. Rev. B 87, 144105-144105 (2013).

23. Zhu, J. et al. Superconductivity in topological insulator $\mathrm{Sb}_{2} \mathrm{Te}_{3}$ induced by pressure. Sci. Rep. 3, 2016 (2013).

24. Liu, B. et al. Pressure induced semiconductor-semimetal transition in $\mathrm{WSe}_{2}$. J. Phys. Chem. C 114, 14251-14254 (2010).

25. Proctor, J. E. et al. High-pressure Raman spectroscopy of graphene. Phys. Rev. B 80, 073408 (2009)

26. Jayaraman, A. Diamond anvil cell and high-pressure physical investigations. Rev. Mod. Phys. 55, 65-108 (1983).

27. Ashcroft, N. W. \& Mermin, N. D. Solid State Physics (Rinehart and Winston, 1976).

28. Yue, Q. et al. Mechanical and electronic properties of monolayer $\mathrm{MoS}_{2}$ under elastic strain. Phys. Lett. A 376, 1166-1170 (2012).
29. Lee, C. et al. Anomalous lattice vibrations of single- and few-layer $\mathrm{MoS}_{2}$. ACS Nano 4, 2695-2700 (2010).

30. Livneh, T. \& Sterer, E. Resonant Raman scattering at exciton states tuned by pressure and temperature in $2 \mathrm{H}-\mathrm{MoS}_{2}$. Phys. Rev. B 81, 195209-195209 (2010).

31. Molina-Sánchez, A. \& Wirtz, L. Phonons in single-layer and few-layer $\mathrm{MoS}_{2}$ and $\mathrm{WS}_{2}$. Phys. Rev. B 84, 155413 (2011).

32. Nicolle, J. et al. Pressure-mediated doping in graphene. Nano Lett. 11, 3564-3568 (2011).

33. Conley, H. J. et al. Bandgap engineering of strained monolayer and bilayer $\mathrm{MoS}_{2}$. Nano Lett. 13, 3626-3630 (2013).

34. Mittal, R. et al. Phonon softening and pressure-induced phase transitions in quartz structured compound, $\mathrm{FePO}_{4}$. Preprint at http://arxiv.org/abs/0903.1550 (2009).

35. Hearn, C. J. Phonon softening and the metal-insulator transition in $\mathrm{VO}_{2}$. J. Phys. C Solid State Phys. 5, 1317 (1972).

36. Yan, J. et al. Observation of anomalous phonon softening in bilayer graphene. Phys. Rev. Lett. 101, 136804 (2008).

37. Huang, M. et al. Phonon softening and crystallographic orientation of strained graphene studied by Raman spectroscopy. Proc. Natl Acad. Sci. USA 106, 7304-7308 (2009).

38. Kudo, K. et al. Giant phonon softening and enhancement of superconductivity by phosphorus doping of $\mathrm{BaNi}_{2} \mathrm{As}_{2}$. Phys. Rev. Lett. 109, 097002 (2012).

39. Bera, A. et al. Sharp Raman anomalies and broken adiabaticity at a pressure induced transition from band to topological insulator in $\mathrm{Sb}_{2} \mathrm{Se}_{3}$. Phys. Rev. Lett. 110, 107401 (2013).

40. Kam, K. K. \& Parkinson, B. A. Detailed photocurrent spectroscopy of the semiconducting group VIB transition metal dichalcogenides. J. Phys. Chem. 86, 463-467 (1982).

41. Perdew, J. P. \& Zunger, A. Self-interaction correction to density-functional approximations for many-electron systems. Phys. Rev. B 23, 5048-5079 (1981).

42. Heyd, J. et al. Hybrid functionals based on a screened Coulomb potential. J. Chem. Phys. 118, 8207-8215 (2003).

43. Hedin, L. New method for calculating the one-particle green's function with application to the electron-gas problem. Phys. Rev. 139, A796-A823 (1965).

44. Choi, W. et al. High-detectivity multilayer $\mathrm{MoS}_{2}$ phototransistors with spectral response from ultraviolet to infrared. Adv. Mater. 24, 5832-5836 (2012).

45. Von Dreele, R. B. \& Larson, A. C. General structure analysis system. Regents of the University of California (2001).

46. Guo, H. et al. High pressure effect on structure, electronic structure, and thermoelectric properties of $\mathrm{MoS}_{2}$. J. Appl. Phys. 113, 0137091-0137097 (2013).

47. Kobayashi, H. et al. Pressure-induced semiconductor-metal-semiconductor transitions in FeS. Phys. Rev. B 63, 115203-1-115203-7 (2001).

48. Feinleib, J. \& Paul, W. Semiconductor-to-metal transition in $\mathrm{V}_{2} \mathrm{O}_{3}$. Phys. Rev. 155, 841-850 (1967).

49. Berglund, C. N. \& Guggenheim, H. J. Electronic properties of $\mathrm{VO}_{2}$ near the semiconductor-metal transition. Phys. Rev. 185, 1022-1033 (1969).

50. Aksoy, R. et al. X-ray diffraction study of molybdenum disulphide to $38.8 \mathrm{GPa}$. J. Phys. Chem. Solids 67, 1914-1917 (2006).

51. Schroder, D. K. Semiconductor material and device characterization (John Wiley \& Sons, 2006).

52. Hammersley, A. P. et al. Two-dimensional detector software: from real detector to idealised image or two-theta scan. High Pressure Res. 14, 235-248 (1996).

53. Meade, C. \& Jeanloz, R. Static compression of $\mathrm{Ca}(\mathrm{OH})_{2}$ at room temperature: observations of amorphization and equation of state measurements to 10.7 GPa. Geophys. Res. Lett. 17, 1157-1160 (1990).

54. Liu, H. et al. Static compression of $\alpha-\mathrm{Fe}_{2} \mathrm{O}_{3}$ : linear incompressibility of lattice parameters and high-pressure transformations. Phys. Chem. Minerals 30, 582-588 (2003).

55. Kresse, G. \& Hafner, J. Ab initio molecular dynamics for liquid metals. Phys. Rev. B 47, 558-561 (1993).

56. Kresse, G. \& Joubert, D. From ultrasoft pseudopotentials to the projector augmented-wave method. Phys. Rev. B 59, 1758-1775 (1999).

57. Blöchl, P. E. Projector augmented-wave method. Phys. Rev. B 50, 17953-17979 (1994).

58. Perdew, J. P. et al. Generalized gradient approximation made simple. Phys. Rev. Lett. 77, 3865-3868 (1996).

59. Grimme, S Semiempirical GGA-type density functional constructed with a long-range dispersion correction. J. Comput. Chem. 27, 1787-1799 (2006).

60. Gonze, X. \& Lee, C. Dynamical matrices, Born effective charges, dielectric permittivity tensors, and interatomic force constants from density-functional perturbation theory. Phys. Rev. B 55, 10355-10368 (1997).

61. Togo, A. et al. First-principles calculations of the ferroelastic transition between rutile-type and $\mathrm{CaCl}_{2}$-type $\mathrm{SiO}_{2}$ at high pressures. Phys. Rev. B 78, 134106 (2008).

62. Ziman, J. M. Principles of the Theory of Solids (Cambridge University Press, 1972).

63. Madsen, G. K. H. \& Singh, D. J. BoltzTraP. A code for calculating bandstructure dependent quantities. Comput. Phys. Commun. 175, 67-71 (2006). 
64. Boyer, L. L. Symmetrized Fourier method for interpolating band-structure results. Phys. Rev. B 19, 2824-2830 (1979).

65. El-Mahalawy, S. H. \& Evans, B. L. Temperature dependence of the electrical conductivity and hall coefficient in $2 \mathrm{H}-\mathrm{MoS}_{2}, \mathrm{MoSe}_{2}, \mathrm{WSe}_{2}$, and $\mathrm{MoTe}_{2}$. Phys. Status Solid. B 79, 713-722 (1977).

\section{Acknowledgements}

Research at The University of Texas at Austin was supported by the Energy Frontier Research in Extreme Environments (EFree) and a Young Investigator Award (D.A.) from the Defense Threat Reduction Agency (DTRA). Research at the Chinese Academy of Sciences was supported by National Science Foundation (NSF) and Ministry of Science and Technology (MOST) of China through research projects. Research at Indian Institute of Science was supported by National Program on Micro and Smart Systems (NpMASS) PARC No. 1:22 and DST Nanomission. We acknowledge High Pressure Collaborative Access Team (HPCAT) and GeoSoilEnviroCARS (GSECARS) of the Advanced Photon Source (APS), Argonne National Laboratory for use of their facilities. We would like to thank the Supercomputer Education and Research Centre and Materials Research Centre, Indian Institute of Science for providing the required computational facilities necessary for this work. We also thank J. Wozniak and Texas Advanced Computing Center (TACC) for their help with the 3D rendering.

\section{Author contributions}

A.P.N. initiated this collaborative research project. J.Z., J.L., X.W. and J.-F.L. designed the experiment. A.P.N, D.A, J.Z., J.L. and X.W developed the measurement setup and performed the measurements. D.A., S.B., T.P. and A.K.S. conducted the theoretical modelling and correlation with experimental data. D.A. led the writing of the paper. All the authors participated in the discussion of the results and the whole project was supervised by A.K.S., D.A. and J.-F.L.

\section{Additional information}

Supplementary Information accompanies this paper at http://www.nature.com/ naturecommunications

Competing financial interests: The authors declare no competing financial interests.

Reprints and permission information is available online at http://npg.nature.com/ reprintsandpermissions/

How to cite this article: Nayak, A. P. et al. Pressure-induced semiconducting to metallic transition in multilayered molybdenum disulphide. Nat. Commun. 5:3731 doi: 10.1038/ncomms4731 (2014). 